\title{
Numerical optimization of integrating cavities for diffraction-limited millimeter-wave bolometer arrays
}

\author{
Jason Glenn, Goutam Chattopadhyay, Samantha F. Edgington, Andrew E. Lange, \\ James J. Bock, Philip D. Mauskopf, and Adrian T. Lee
}

\begin{abstract}
Far-infrared to millimeter-wave bolometers designed to make astronomical observations are typically encased in integrating cavities at the termination of feedhorns or Winston cones. This photometer combination maximizes absorption of radiation, enables the absorber area to be minimized, and controls the directivity of absorption, thereby reducing susceptibility to stray light. In the next decade, arrays of hundreds of silicon nitride micromesh bolometers with planar architectures will be used in ground-based, suborbital, and orbital platforms for astronomy. The optimization of integrating cavity designs is required for achieving the highest possible sensitivity for these arrays. We report numerical simulations of the electromagnetic fields in integrating cavities with an infinite plane-parallel geometry formed by a solid reflecting backshort and the back surface of a feedhorn array block. Performance of this architecture for the bolometer array camera (Bolocam) for cosmology at a frequency of $214 \mathrm{GHz}$ is investigated. We explore the sensitivity of absorption efficiency to absorber impedance and backshort location and the magnitude of leakage from cavities. The simulations are compared with experimental data from a room-temperature scale model and with the performance of Bolocam at a temperature of $300 \mathrm{mK}$. The main results of the simulations for Bolocam-type cavities are that (1) monochromatic absorptions as high as $95 \%$ are achievable with $<1 \%$ cross talk between neighboring cavities, (2) the optimum absorber impedances are $400 \Omega$ sq, but with a broad maximum from $\sim 150$ to $\sim 700 \Omega /$ sq, and (3) maximum absorption is achieved with absorber diameters $\geq 1.5 \lambda$. Good general agreement between the simulations and the experiments was found. (C) 2002 Optical Society of America
\end{abstract}

OCIS codes: $040.1240,040.3060,030.1940$.

\section{Introduction}

Infrared and millimeter-wave bolometers designed for astronomical observations typically consist of thermistors bonded to two-dimensional radiation absorbers ${ }^{1,2}$ encased in integrating cavities behind feedhorns. The combination of feedhorns and cavities minimizes absorber areas (and therefore bolometer

J. Glenn (jglenn@casa.colorado.edu) is with the Center for Astrophysics and Space Astronomy, UCB-389, University of Colorado, Boulder, Colorado 80309. G. Chattopadhyay, S. F. Edgington, and A. E. Lange are with the Department of Physics, MC 59-33, California Institute of Technology, Pasadena, California 91125. J. J. Bock is with the Jet Propulsion Laboratory, MS 169-327, 4800 Oak Grove Drive, Pasadena, California 91109. P. D. Mauskopf is with the Department of Physics and Astronomy, University of Wales, Cardiff, UK. A. T. Lee is with the Department of Physics, University of California, Berkeley, Berkeley, California 94720 .

Received 9 February 2001; revised manuscript received 7 August 2001.

0003-6935/02/010136-07\$15.00/0

(C) 2002 Optical Society of America heat capacities and thermal time constants), controls the directivity of absorption, and reduces susceptibility to stray light. The figure of merit for bolometers is $\mathrm{NEP}^{-1 / 2}$, where the NEP is the noise-equivalent power in $\mathrm{W} \mathrm{Hz}^{-1 / 2}$, and $\tau \propto C$ is the thermal time constant (typically a few milliseconds) for a heat capacity $C$. $\quad \mathrm{NEP}^{-1 / 2}$ is minimized for optimal bolometers. Stray light is particularly problematic for the highly sensitive (NEPs approaching $10^{-19} \mathrm{~W} \mathrm{~Hz}^{-1 / 2}$ ), subkelvin (100-mK to $300-\mathrm{mK})$ bolometers used for observations of faint sources, such as cosmic microwave background anisotropies and the thermal dust emission from distant galaxies.

The angular response and throughput of Winston cones coupled to integrating cavities are understood in the geometrical optics limit. ${ }^{3,4}$ Recently, bolometers have been used in submillimeter and millimeter-wave applications for astronomy in the single-mode (diffraction) limit5,6, motivating an optimization of bolometric integrating cavities in singlemode systems. Efficient single-mode integrating cavities coupled to scalar feedhorns and rectangular waveguides have already been investigated, ${ }^{7}$ and the 
throughput and angular response of straight-walled conical feedhorns coupled to circular waveguides have been analyzed in the few-mode case. ${ }^{8}$ In this paper we investigate coupling of radiation to bolometers with single-mode circular waveguides and integrating cavities in the geometry afforded by planar arrays of bolometers on contiguous silicon wafers.

A new submillimeter bolometer construction with silicon nitride micromesh absorbers etched into wafers of a silicon substrate lends itself naturally to planar arrays of hundreds of detectors. ${ }^{9}$ The absorbers consist of silicon nitride micromeshes in a spider-web pattern metallized for radiation absorption. The micromeshes reduce heat capacity and cross section to cosmic-ray strikes compared with solid absorbers. They are characterized by a grid spacing that scales with wavelength. Silicon nitride micromesh bolometers are finding widespread use in ground-based, suborbital, and space platforms for astronomy. These arrays will be used for the bolometer array camera, Bolocam, ${ }^{10}$ a ground-based camera for cosmological observations with 144 bolometers, and the spectral photometric imaging receiver (SPIRE) on the Herschel Space Observatory ${ }^{11,12}$ that will have five arrays, totaling 326 bolometers. In both applications, the bolometers are packed hexagonally on planar wafers sandwiched between feedhorn plates and planar backshorts. The feedhorns terminate in circular waveguides followed by integrating cavities formed by the back of the feedhorn plate, the silicon wafer substrate, and the backshort plate. This bolometer array geometry precludes use of ideal integrating cavities with metallic walls forming $4 \pi \mathrm{sr}$ of a perfect reflector.

We used the Hewlett-Packard high-frequency structure simulator (HFSS) ${ }^{13}$ to simulate absorption in integrating cavities. HFSS solves electromagnetic fields in three-dimensional structures with specified boundary conditions employing a finiteelement method. In general, the finite-element method divides the full problem space into thousands of elements and represents the field in each element with a local function. HFSS is a powerful tool for the electromagnetic solutions of complicated threedimensional structures and often renders scalar model measurements unnecessary. HFSS is not practical for structures that are large compared to a wavelength because large amounts of computation time ( $>1$ day) and memory are needed to converge to an accurate solution.

Specifically, we investigated the absorption efficiency as a function of the easily adjustable integrating cavity parameters, including the absorber diameter, the absorber impedance, and absorber placement in the cavity. We also estimated the leakage of radiation out of the cavities. Leakage is not only as a loss, but is also a source of optical cross talk between pixels, thereby enlarging the otherwise diffraction-limited point-spread function of Bolocam. A subset of the results were compared with monochromatic measurements of a room-

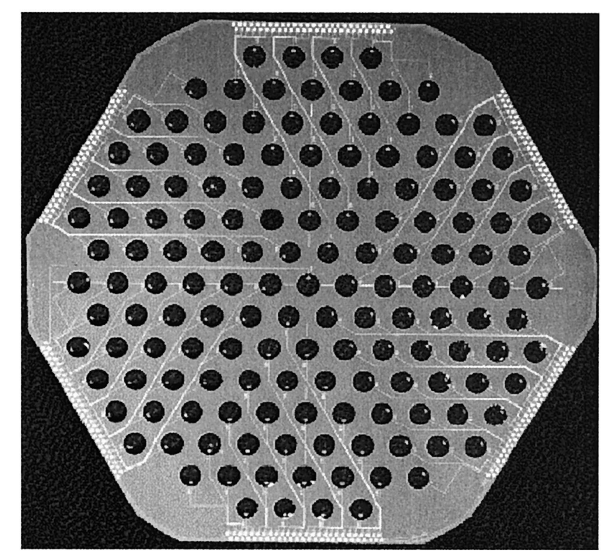

(a)

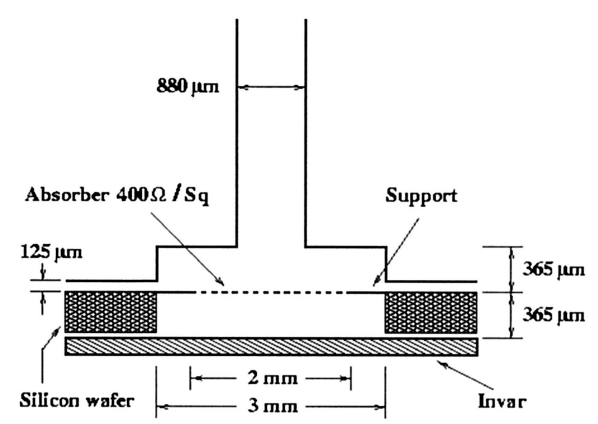

(b)

Fig. 1. (a) Bolocam $(214 \mathrm{GHz})$, manufactured at the Jet Propulsion Laboratory Micro Devices Laboratory, is composed of a silicon wafer $75 \mathrm{~mm}$ in diameter and $400 \mu \mathrm{m}$ thick. It has $151 \mathrm{mi}$ cromesh absorbers etched into it and semiconductor thermistors attached to each absorber. The absorber meshes and thermistors are too small to see in this rendering. (b) Geometry of a single 214-GHz bolometer integrating cavity seen in cross section edgeon. There are 144 integrating cavities in Bolocam, one for each bolometer in the array. The cavity is circularly symmetric about the axis of the waveguide. The aluminum feedhorn block (on top, containing the hollow waveguide) and Invar backshort plate were modeled as perfectly reflecting boundaries. There is no gap between the Invar backshort plate and the silicon wafer substrate. The absorber was modeled as an infinitely thin sheet with the appropriate surface impedance. The vacuum gap between the silicon wafer and the feedhorn block leads to adjacent cavities and was modeled as a radiation-absorbing boundary. The wafer used for cryogenic testing in Bolocam was $200 \mu \mathrm{m}$ thick, so cylindrical pockets were milled in the Invar backing plate to form the $\lambda / 4$ backshorts.

temperature scale model and broadband measurements with Bolocam.

\section{Description of Numerical Simulations}

A picture of the Bolocam bolometer array is is shown in Fig. 1(a), and the integrating cavity configuration is shown in Fig. 1(b). The dimensions of this configuration are suitable for $214 \mathrm{GHz}(\lambda=1.4 \mathrm{~mm})$, but the results are directly generalizable when we scale the dimensions by $\lambda$. The SPIRE photometers (250-, $350-$, and $500-\mu \mathrm{m}$ center wavelengths) share this basic geometry. An important utility of bolometers is 
the frequency independence of absorption (up to wavelengths of the order of the absorber size). Bandpasses in bolometric systems are typically defined by bandpass filters in the optical path preceding the feedhorns. It is desirable to achieve high absorption efficiency across the bandpass, although it is clearly not possible to achieve uniform absorption across wide bands with resonant cavities. Our strategy was to tune the bolometers, cavities, and feedhorns for optimum absorption at the band centers and investigate the absorption at other frequencies in the band. Bolocam will have bandpasses of $\Delta v / v \sim 0.15$, whereas the SPIRE photometer bandpasses will be $\Delta v / v \sim 0.33$. SPIRE will also have two Fourier-transform spectrometers with total continuous coverage of $200-670 \mu \mathrm{m}$. The bandpasses will each cover an octave of frequency and may require more complicated cavities, which will be a topic of future study.

Ideally, the walls of an integrating cavity would be uniformly reflecting. However, a vacuum gap between the wafer and the feedhorn plate is necessary to prevent mechanical damage to the wafer. Consequently, some radiation will escape through the vacuum gap. Furthermore, the lower cavity sidewalls formed by the silicon wafer have an index of refraction of $n=3.4$. For a wave with normal incidence, $30 \%$ of the radiation would be reflected back into the cavity and $70 \%$ would be transmitted into the silicon. Radiation can reach the sidewalls by diffraction or multiple reflections. Because most of this radiation will not be normally incident on the sidewalls, more than $30 \%$ of the radiation will be reflected back into the cavity.

Absorption should be maximized with the absorber placed in the center of the cavity, a distance of $\lambda_{c} / 4$ from both the waveguide termination and the backshort plane, where $\lambda_{c}$ is the center of the bandpass. This was the starting point for our simulations. The center-to-center detector spacing of $5 \mathrm{~mm}$ was chosen to maximize the number of pixels in the Bolocam focal plane while retaining relatively efficient feedhorns. ${ }^{12}$ The feedhorns have $1.2 f \lambda_{c}$ entrance apertures, where $f$ is the focal ratio of the final optics ( $f / 3$ for Bolocam). The absorbers were $2.0 \mathrm{~mm}$ in diameter, with $\mathrm{Si}_{3} \mathrm{~N}_{4}$ support legs $0.5 \mathrm{~mm}$ long providing thermal isolation from the silicon wafer, which is heat stationed to the $300-\mathrm{mK}$ bath. The cavity dimensions were therefore $2.14 \lambda_{c}$ in diameter and $\lambda_{c} / 2$ in height (along the axis of wave propagation).

An initial guess for the absorber impedance to maximize absorption is motivated by a straightforward calculation in free space. In free space, the absorption coefficient is given by

$$
A=1-R-T=\frac{4 g_{a} Z_{0} l}{\left(2+g_{a} Z_{0} l\right)^{2}},
$$

where $R$ is the absorber reflection coefficient, $T$ is the transmission coefficient, $l$ is the absorber thickness, $Z_{0}$ is the impedance of free space $(377 \Omega / \mathrm{sq})$, and $g_{a}=$ $1 / Z_{a} l$ is the absorber conductivity. The maximum absorption coefficient is $A=0.5$ for an absorber with an impedance of $Z_{a}=189 \Omega / \mathrm{sq}$, which is not the same as the impedance that minimizes reflection when we match $Z_{a}$ to the impedance of free space. So Eq. (1) shows that even a perfect absorber can absorb only half of the incident radiation in the absence of a reflecting backshort. Because the impedance of the cavity will be larger than the impedance of free space, the simulations should yield an optimum absorber impedance $Z_{a}>189 \Omega$.

Our objectives of the simulations were twofold: to maximize the optical coupling efficiency of the cavity by optimizing the absorber impedance and diameter and to determine the amount of leakage out of cavities, which would cause cross talk. We formed the 3 -mm diameter of the upper part of the cavity in Fig. 1 by machining cylindrical pockets into the back of the feedhorn array to match the 3-mm total diameter of the absorber and support legs. The cavity was at the termination of the $880-\mu \mathrm{m}$-diameter circular waveguide that provided a low-frequency cutoff at $200 \mathrm{GHz}$ for the dominant $\mathrm{TE}_{11}$ mode wave. We took advantage of the electromagnetic symmetry of the structure by simulating only one half of the cavity, which reduced the simulation time by almost half. H-wall symmetry was applied such that the electric field was tangential to the midplane of the waveguide and cavity and the magnetic field was normal to the plane. The $125-\mu \mathrm{m}$ vacuum gap in the structure was simulated by a radiation-absorbing boundary (i.e., radiation was permitted to leave the cavity through the gap to simulate the absence of a boundary). A single port was defined at the feedhorn end of the circular guide and was excited with a $\mathrm{TE}_{11}$ mode wave. The absorber was simulated as an infinitely thin conducting sheet parallel to and at a distance of $\lambda_{c} / 4$ from the backshort. A simulation was also performed with a finite-thickness absorber to test the infinitely thin approximation. The results were the same, but the simulation took substantially more time because the fields had to be solved for within the absorber.

Figure 2 shows the details of the drawing used for the HFSS simulation. Radiation can leak out of the cavity by reflecting out of port 1 or by transmitting through the vacuum gap and silicon wafer substrate. To measure the power absorbed by the bolometer and the power leakage out of the cavity, we performed simulations with and without the absorber present. For a cavity with no absorber, no leakage through the silicon wafer or vacuum gap, and no power converted to other modes, all the power would be reflected back to port $1\left(S_{11}=1\right)$. Because we are restricted to measuring only the reflected power $S_{11}$, the following assumptions were necessary: (1) The presence of the absorber does not affect the power leakage through the vacuum gap and silicon wafer, and (2) the higher-order modes generated as the wave passes into the cavity are evanescent. With these two assumptions, performing simulations with and without the absorber present allowed us to separate the power absorbed by the bolometer, the power that 


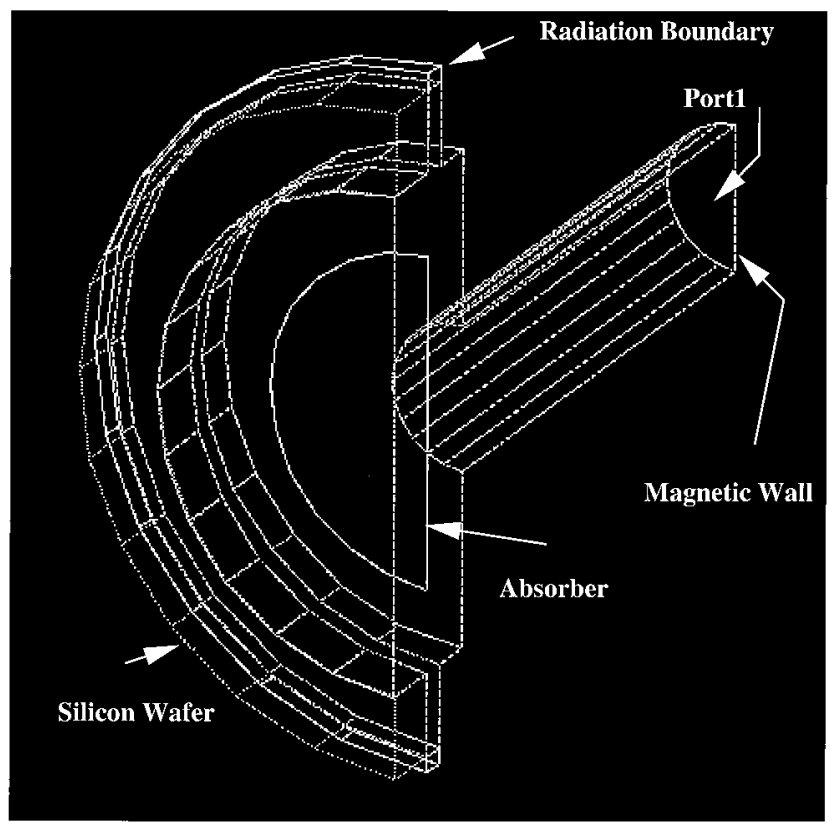

Fig. 2. Configuration used for the HFSS simulations showing the ports and the boundaries in an oblique view. A magnetic symmetry wall was employed to take advantage of the structural symmetry and reduce the numerical computation time.

leaked out the vacuum gap and silicon wafer, and the power that reflected out of the waveguide. The first assumption can overestimate the power that leaked out of the gap and silicon with the absorber present, thereby causing the power absorbed measured in this way to be slightly underestimated. For the optimum absorption cases reported in this paper, this is an underestimation of $\leq 4 \%\left(1-S_{11}^{2}\right.$ with no absorber present and the presence of an absorber would decrease leakage; see Subsection 3.A). Higher-order modes exiting the cavity through port 1 would not be detected, leading to an overestimation of the absorbed power. However, the simulations them- selves showed that the higher-order modes generally were evanescent and therefore that assumption 2 was valid.

\section{Results and Discussion}

\section{A. Numerical Simulations}

The results of the numerical simulations and experimental tests are summarized in Table 1. The simulations were performed in an iterative fashion to optimize one parameter at a time. First, $S_{11}$ was measured for the initial cavity design with a range of absorber impedances and diameters to find the configuration with the smallest return loss, and hence greatest absorption. The optimum absorber impedance with an absorber diameter of $2.1 \lambda_{c}=3.0 \mathrm{~mm}$ (which corresponds to the Bolocam configuration) was found to be $400 \Omega / \mathrm{sq}$. This differs from the optimum $200 \Omega$ /sq in free space. However, the maximum was broad, ranging from 150 to $700 \Omega$ /sq (Fig. 3 ). Next, with the impedance set to $400 \Omega / \mathrm{sq}$, the diameter was varied from $1.0 \mathrm{~mm}\left(0.7 \lambda_{c}\right)$ to $3.0 \mathrm{~mm}$ $\left(2.1 \lambda_{c}\right)$. The return loss was found to be the same for absorber diameters down to $2.0 \mathrm{~mm}$, then increased with further decreasing absorber diameters (Fig. 4).

The instantaneous electric field magnitude $|\mathbf{E}|$ for the simulation with $Z_{a}=400 \Omega / \mathrm{sq}$ and a $3.0-\mathrm{mm}$ absorber diameter are shown in two views in Fig. 5. The particular instant in the simulation portrayed in Fig. 5(a) was chosen because it clearly shows the wave being effectively absorbed by the bolometer. Figure 5(b) shows the orthogonal distribution of the electric field in the cavity projected parallel to the plane of the absorber. Because the waveguide diameter is so much smaller than the cavity diameter, the electromagnetic fields are concentrated toward the center of the cavity. $|\mathbf{E}|$ drops nearly linearly from the center to the edges, and little power makes it to the edges of the cavity where it can escape. Because it is advantageous to minimize the absorber area to minimize the detector thermal time constant, the

Table 1. Summary of HFSS Simulations and Experimental Results

\begin{tabular}{lccc}
\hline \multicolumn{1}{c}{ Parameter } & HFSS & Bolocam & Scale Model \\
\hline Frequency & $214 \mathrm{GHz}$ & $205-233 \mathrm{GHz}$ & $75-110 \mathrm{GHz}$ \\
Optimum $Z_{a}$ & $400 \Omega / \mathrm{sq}$ & - & - \\
$Z_{a}$ range for $S_{11}{ }^{2}$ less than $-20 \mathrm{~dB}(A>0.99)$ & $150-700 \Omega / \mathrm{sq}$ & - & - \\
Optimum absorber diameter & $\geq 1.5 \lambda_{c}$ & - & - \\
$S_{11}{ }^{2}$ without absorber & $0.96(-0.2 \mathrm{~dB})$ & - & $0.02(-15 \mathrm{~dB})$ \\
$S_{11}{ }^{2}$ with absorber & $0.01(-19 \mathrm{~dB})$ & $0.15 \pm 0.10(-8.2 \mathrm{~dB})$ & - \\
Power leakage $^{a, b}(L)$ & $0.04(-14 \mathrm{~dB})$ & $0.85 \pm 0.10(-0.7 \mathrm{~dB})$ & $0.97(-0.1 \mathrm{~dB})$ \\
Power absorption $^{\alpha, c}(A)$ & $0.95(-0.2 \mathrm{~dB})$ & $<0.03($ less than $-15.2 \mathrm{~dB})$ & - \\
Cross-talk power $_{\Delta v / v \text { range }}$ for $S_{11}{ }^{2}$ less than $-10 \mathrm{~dB}(A>0.90)$ & $\leq 0.01^{d}(-20 \mathrm{~dB})$ & - & 0.2 \\
Power absorption $^{c}(A)$ with $\lambda_{c} / 2$ shorts & - & - & $0.50(-3.0 \mathrm{~dB})$ \\
\hline
\end{tabular}

${ }^{a}$ Calculated with the optimum cavity configuration.

${ }^{b}$ For the HFSS simulations, the leakage was derived from the return loss measured with no absorber present: $L=1-S_{11}{ }^{2}$. For the Bolocam measurements, the absorbed power rather than the return loss was measured, so the leakage was defined as $L=1-A$.

'Absorption $A=1-S_{11}{ }^{2}-L$.

${ }^{d}$ For a hexagonal pack, the cross talk from one detector to an adjacent detector is the leakage $L$ divided by 6 .

${ }^{e} S_{11}{ }^{2}$ is less than $-10 \mathrm{~dB}$ because we assume there is no loss from the scale model cavity. 


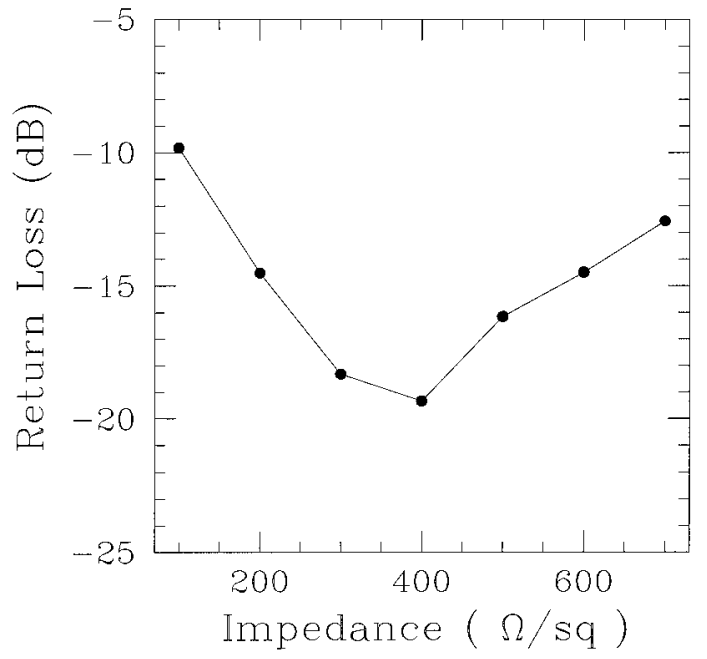

Fig. 3. Simulated $\mathrm{TE}_{11}$ reflection (return loss) $S_{11}$ at port 1 as a function of absorber impedance. The absorber diameter was fixed at $2.0 \mathrm{~mm}$. Maximum absorption occurs for a surface impedance of $400 \Omega / \mathrm{sq}$.

simulations indicate that it is best to limit the absorber area to $\sim 1.5 \lambda_{c}$ in this single-mode configuration.

With the optimum absorber impedance and diameter, the power leakage out of the cavity and power absorbed by the bolometer were measured. With no absorber present (assuming no power is converted to higher-order modes and reflected out of the waveguide), the leakage $L$ is simply given by $L=1-S_{11}{ }^{2}$. For the observed $S_{11}{ }^{2}=0.96$, the leakage was $L=0.04$, normalized to an input power of unity (hereafter all powers are referred to the normalized unity input power). Therefore, because the detectors in all the applications will be

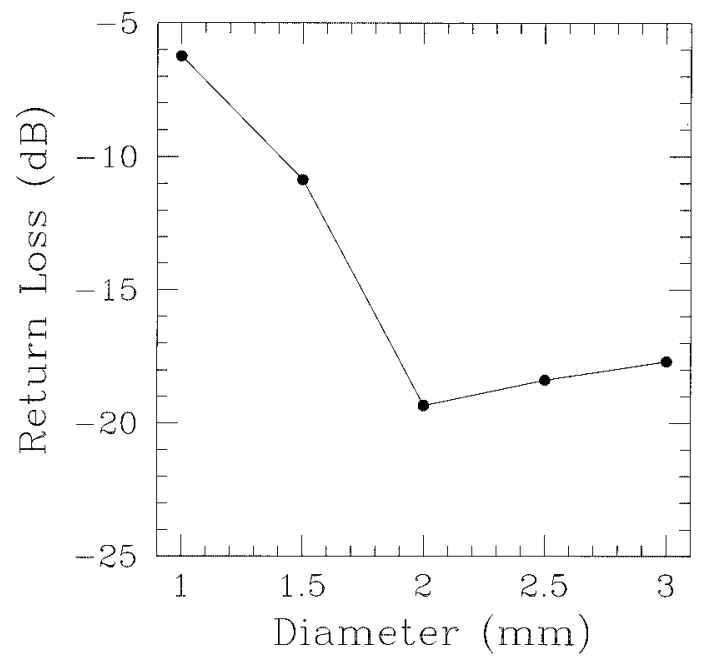

Fig. 4. Simulated reflection (return loss) $S_{11}$ at port 1 of the 3.0-mm-diameter cavity as a function of absorber diameter. The absorber impedance was fixed at $400 \Omega /$ sq. For this cavity configuration, an absorber diameter of $2.0 \mathrm{~mm}$ affords a low return loss and a minimum absorber area.

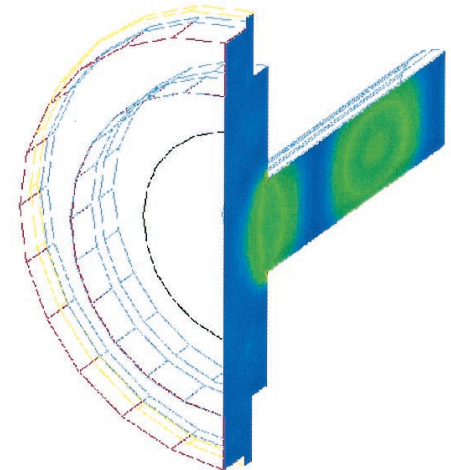

(a)

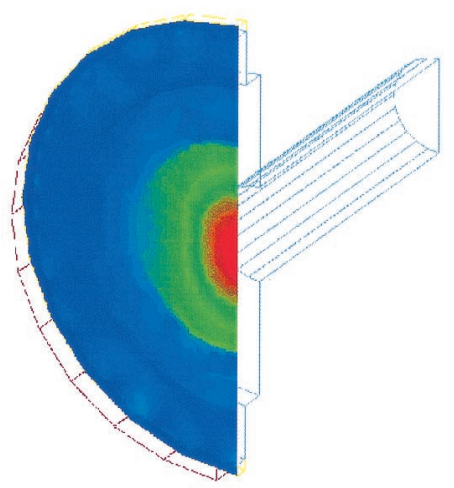

(b)

Fig. 5. Instantaneous electric field strengths $|\mathbf{E}|$ from the simulations. Red is maximum field strength and blue is minimum. The color stretches are linear. (a) $\mathrm{TE}_{11}$ mode propagating in the waveguide, with a cut along the waveguide axis. Absorption by the bolometer spanning the center of the cavity is apparent. (b) Distribution of the field strength in the plane of the absorber. $|\mathbf{E}|$ falls nearly linearly from the center of the cavity (waveguide axis) to the edges, which are defined by the silicon and upper cavity walls.

layed out on a hexagonal grid with six nearest neighbors, the cross talk between adjacent detectors should be $<0.01$. With the absorber in place, the simulated return loss was $S_{11}{ }^{2}=0.01$. The absorbed power for the optimum cavity is $A=1-$ $S_{11}{ }^{2}-L$, where $L$ replaces $T$ when we apply Eq. (1) to the entire cavity. Therefore, monochromatically, the highest attainable fractional absorbed power with the plane-parallel cavity structure is 0.95 .

A convergence criterion of $\Delta S_{11} \leq 0.008$ was applied to all the simulations. Although this convergence criterion is not strictly an uncertainty (and therefore not listed in Table 1), it can be used to estimate the numerical sensitivities of the parameters derived from $S_{11}$ to $\Delta S_{11} \leq 0.008$. Applying straightforward error propagation to the derived parameters yields $S_{11}$ (without absorber) $=0.96 \pm 0.02$, $S_{11}$ (with absorber) $=0.01 \pm 0.002, L=0.04 \pm 0.02$, $A=0.95 \pm 0.02$, and a cross talk of $0.007 \pm 0.003$ between adjacent bolometers. 


\section{B. $90-\mathrm{GHz}$ Room-Temperature Scale Model}

Generally, bolometric applications are broadband, whereas HFSS simulations are monochromatic by nature. Performing multiple monochromatic HFSS simulations would be time-consuming because many simulations would have to be performed to sample the bandpass. Furthermore, the simulations become computationally intensive as the frequency approaches the waveguide cutoff frequency. Consequently, we measured the bandwidth of the entire configuration by measuring $S_{11}$ with a $90-\mathrm{GHz}$ scale model and a network analyzer and by measuring the absorption efficiency with Bolocam over a $\Delta v / v=0.14$ bandpass. These tests also served to validate the results of the simulations.

We made the scale model measurements by exciting $\mathrm{TE}_{11}$ mode waves over a range in frequencies from 77 to $109 \mathrm{GHz}$ in a waveguide coupled to a cavity and measuring the return loss in the same mode. These measurements were therefore subject to the same systematic effect as the simulations because higher-order modes could be created and propagate out of the waveguide, escaping detection. This could lead to a slight overestimation of the absorption. To the extent that the scale model replicates the simulations, the higher-order modes are likely evanescent and do not contribute substantial power leakage.

The configuration differed somewhat from the simulated Bolocam cavities because a single bolometer was used. The cavity walls were nearly completely sealed, with holes only for the bolometer wires and waveguide. In principle, this should lead to slightly higher absorption for the scale model than for the infinite cavity structure in the simulations. Two silicon nitride micromesh absorbers of 4.8- and 5.6-mm diameters were used. These correspond to diameters of $1.5 \lambda_{c}$ and $1.7 \lambda_{c}$, so there should be little difference in performance for the two detectors. The surface impedances were $Z_{a} \sim 200 \Omega /$ sq, close to the absorption peak. The results are shown in Fig. 6 . The absorption is greater than 0.90 across a band of $\Delta v / v=0.2$, roughly centered on the $90-\mathrm{GHz}$ scale model design frequency. Furthermore, the scale model yielded $0.97 \pm 0.03$ absorption at the band center, close to the 0.95 predicted by the simulations. The curves for the 5.6-mm-diameter absorber are shifted to slightly lower frequencies than the curves for the 4.8-mm absorbers. This is consistent with the expectation that larger absorbers have slightly better absorption at the longer wavelengths.

It is interesting to investigate the expected performance for a cavity design with optimal absorber impedance and diameter but nonoptimal backshort location. For the worst case, we performed a simulation with the absorber located $\lambda_{c} / 2$ from the waveguide termination and backshort, thereby creating destructive interference in the electric field at the detector. The absorption predicted by the simulations was $A=0.36$, indicating that the cavity behaved not only in a resonant fashion, but also somewhat in the true sense of an integrating cavity.

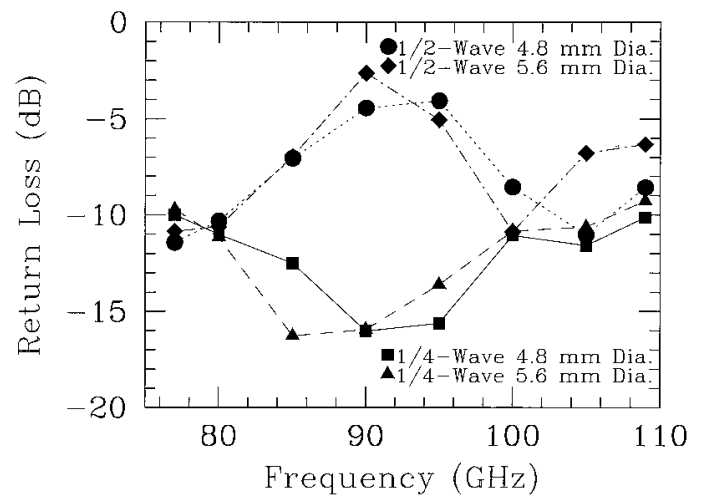

Fig. 6. Scale model $90-\mathrm{GHz}$ measurements of return loss as a function of frequency for a 4.8-mm-diameter absorber $\lambda_{c} / 4$ from the waveguide termination and backshort, $5.6-\mathrm{mm}$ absorber $\lambda_{c} / 4$ from the waveguide termination and backshort, $4.8-\mathrm{mm}$ absorber $\lambda_{c} / 2$ from the waveguide termination and backshort, and 5.6-mm absorber $\lambda_{c} / 2$ from the waveguide termination and backshort. It is clear that, with the absorbers present, the cavities are tuned for absorption with $\lambda_{c} / 4$ spacings and tuned for reflection with $\lambda_{c} / 2$ spacings.

An identical test with the scale model yielded an absorption of $A=0.50$, with the return loss peaked at $90 \mathrm{GHz}$. The discrepancy between the simulations and the scale model results seems to be real. A likely explanation is that the leakage out of the nonoptimal cavity in the simulations is worse than in the scale model cavity with nearly solid walls, especially because the structure is not resonant.

\section{Cryogenic Tests with Bolocam}

For the absorption test with Bolocam, we used the optical efficiency measurement technique described by Church et al.14 Two blackbody loads with temperatures of 77 and $293 \mathrm{~K}$ were alternately placed in front of three Bolocam feedhorns to completely fill their beams. The difference in the optical power absorbed with each load was measured from thermistor electrical load curves. The measured differential power was then compared with the theoretical power incident on the detectors. The known losses of the optical elements and the throughputs derived from beam maps and measured spectral bandpasses were included. The mean absorption was $A=0.85 \pm$ 0.10 , where the uncertainty is representative of the dispersion between the three detectors. This result is in good agreement with the simulations.

Cross talk between adjacent pixels was also measured with Bolocam. We illuminated the bolometers by placing a $1070 \mathrm{~K}$ chopped blackbody at an image of the focal plane. An aperture smaller than the beam size permitted the blackbody source to be centered on a single detector at a time. The signal in adjacent unilluminated detectors was then measured, yielding an upper limit of pixel-to-pixel cross talk of $<0.03$, consistent with the $<0.01$ predicted by the simulations. The actual cross talk could be substantially less than 0.03 because the measurement was limited by beam overlap at the focal-plane image. 


\section{Conclusions}

In this paper we reported on numerical simulations performed with HFSS to investigate the absorption of bolometric absorbers in integrating cavities as a function of cavity geometry and absorber impedance. Bolometer arrays in planar architectures for astronomy will find widespread use in ground-based and orbital platforms in the coming decade, motivating a careful optimization of integrating cavities. The bolometer and integrating cavity configuration of Bolocam, a millimeter-wave bolometer camera for cosmology, was used as the baseline for simulations. Experiments with Bolocam $\left(v_{c}=214 \mathrm{GHz}, \Delta v / v=\right.$ 0.14 , and $300-\mathrm{mK}$ bolometers) and a $90-\mathrm{GHz}$ singledetector scale model were used for comparison to the simulations.

Together, the tests show that we can obtain an efficiency factor of three by placing the absorber at a constructive interference maximum. An absorber located $\lambda / 4$ from the waveguide termination and backshort can theoretically achieve a monochromatic absorption of 0.95 , even with only partially reflecting cavity sidewalls. The optimum absorber impedance is $400 \Omega / \mathrm{sq}$. However, the absorption is $>0.90$ for impedances from 150 to $700 \Omega$ /sq. For a cavity $2.1 \lambda_{c}$ in diameter, absorption is enhanced little when we make the absorber larger than $1.5 \lambda_{c}$ in diameter, whereas a smaller absorber results in reduced absorption. The leakage of radiation out of the integrating cavities with partially reflecting walls predicted by simulations is $<0.01$, consistent with the experimentally measured $<0.03$. The experimental data indicate that the absorption remains high $(\geq 0.90)$ across bandpasses up to $\Delta v / v \sim 0.2$. We found that a poorly placed absorber with optimum impedance and diameter, located $\lambda_{c} / 2$ from the waveguide termination and backshort, has an absorption of $\sim 0.35-0.50$. This implies that the cavity still functions somewhat in the sense of an integrating cavity.

We thank Jonas Zmuidzinas for helpful discussions and Sunil Golwala for critical comments on the manuscript. J. Glenn and A. E. Lange acknowledge support from National Science Foundation (NSF) grant AST-9618798. A. Lee was supported by NSF grant AST-9120005.

\section{References}

1. D. C. Alsop, C. Inman, A. E. Lange, and T. Willbanks, "Design and construction of high-sensitivity infrared bolometers for operation at $300 \mathrm{mK}$," Appl. Opt. 31, 6610-6615 (1992).

2. P. L. Richards, "Bolometers for infrared and millimeter waves," J. Appl. Phys. 76, 1-24 (1994).

3. R. Winston, "Light collection within the framework of geometrical optics," J. Opt. Soc. Am. 60, 245-247 (1970).

4. D. A. Harper, R. H. Hildebrand, R. Stiening, and R. Winston, "Heat trap: an optimized far-infrared field optics system," Appl. Opt. 15, 53-60 (1976).

5. C. R. Cunningham, W. K. Gear, W. D. Duncan, P. R. Hastings, and W. S. Holland, "SCUBA: the submillimeter common-user bolometer array for the James Clerk Maxwell telescope," in Instrumentation in Astronomy VIII, D. L. Crawford and E. R. Craine, eds., Proc. SPIE 2198, 638-649 (1994).

6. R. H. Hildebrand, "Focal plane optics in far-infrared and submillimeter astronomy," Opt. Eng. 25, 323-330 (1986).

7. J. C. Peterson and M. A. Goldman, "Reflectance of broad band waveguide bolometers," Int. J. Infrared Millim. Waves 9, 55-69 (1988).

8. J. A. Murphy and R. Padman, "Radiation patterns of fewmoded horns and condensing lightpipes," Infrared Phys. 31, 291-299 (1991).

9. P. D. Mauskopf, J. J. Bock, H. Del Castillo, W. L. Holzapfel, and A. E. Lange, "Composite infrared bolometers with $\mathrm{Si}_{3} \mathrm{~N}_{4}$ micromesh absorbers," Appl. Opt. 36, 765-771 (1997).

10. J. Glenn, J. J. Bock, G. Chattopadhyay, S. F. Edgington, A. E. Lange, J. Zmuidzinas, P. D. Mauskopf, B. Rownd, and L. Yuen, "Bolocam: a millimeter-wave bolometric camera," in Advanced Technology MMW, Radio, and Terahertz Telescopes, T. G. Phillips, ed., Proc. SPIE 3357, 326-334 (1998).

11. M. Griffin, L. Vigroux, and B. Swinyard, "SPIRE: a bolometer instrument for FIRST," in Advanced Technology MMW, Radio, and Terahertz Telescopes, T. G. Phillips, ed., Proc. SPIE 3357, 404-413 (1998).

12. J. J. Bock, J. Glenn, S. M. Grannan, K. D. Irwin, A. E. Lange, H. G. LeDuc, and A. D. Turner, "Silicon nitride micromesh bolometer arrays for SPIRE," in Advanced Technology MMW, Radio, and Terahertz Telescopes, T. G. Phillips, ed., Proc. SPIE 3357, 297-304 (1998).

13. High-Frequency Structure Simulator (HFSS), version A.04.01, Hewlett-Packard Company, Test and Measurement Organization, P.O. Box 50637, Palo Alto, Calif. 94303-9512.

14. S. E. Church, B. Philhour, A. E. Lange, P. A. R. Ade, B. Maffei, R. Nartallo-Garcia, and M. Dragovan, "A compact highefficiency feed structure for cosmic microwave background astronomy at millimeter wavelengths," in Submillimetre and Far-Infrared Space Instrumentation, E. J. Rolfe, ed., Proceedings of the 30th ESLAB Symposium, ESA SP-388 (European Space Agency, Munich, 1996), pp. 77-80. 